\title{
Using the Waseda Bioinstrumentation System WB-1R to Analyze Surgeon's Performance during Laparoscopy - towards the development of a Global Performance Index -
}

\author{
Massimiliano Zecca ${ }^{1,2}$, Filippo Cavallo ${ }^{3,4}$, Minoru Saito ${ }^{5}$, Nobutsuna Endo ${ }^{5}$, \\ Yu Mizoguchi ${ }^{5}$, Stefano Sinigaglia ${ }^{3,4}$, Kazuko Itoh $^{2,6}$, Hideaki Takanobu ${ }^{2,7,8}$, Giuseppe Megali ${ }^{3,4}$, \\ Oliver Tonet ${ }^{3,4}$, Paolo Dario ${ }^{3,4}$, Andrea Pietrabissa ${ }^{4}$, and Atsuo Takanishi ${ }^{1,2,6,7,9}$
}

\begin{abstract}
Minimally invasive surgery (MIS) has become very common in recent years, thanks to the many advantages it provides for patients. Since it is difficult for surgeons to learn and master this technique, several training methods and metrics have been proposed, both to improve the surgeon's abilities and also to assess his/her skills.

This paper presents the use of the WB-1R (Waseda $\underline{B}$ ioinstrumentation system no. $\underline{1}$ Refined), which was developed at Waseda University, Tokyo, to investigate and analyze a surgeon's movements and performance. Specifically, the system can measure the movements of the head, the arms, and the hands, as well as several physiological parameters. In this paper we present our experiment to evaluate a surgeon's ability to handle surgical instruments and his/her depth perception using a laparoscopic view.

Our preliminary analysis of a subset of the acquired data (i.e. comfort of the subjects; the amount of time it took o complete each exercise; and respiration) clearly shows that the expert surgeon and the group of medical students perform very differently. Therefore, WB-1R (or, better, a newer version tailored specifically for use in the operating room) could provide important additional information to help assess the experience and performance of surgeons, thus leading to the development of a Global Performance Index for surgeons during MIS. These analyses and modeling, moreover, are an important step towards the automatization and the robotic assistance of the surgical gesture.
\end{abstract}

1. Institute for Biomedical Engineering, ASMeW, Waseda University, 513, Wasedatsurumaki-cho, Shinjuku-ku, Tokyo 162-0041 JAPAN Tel: +81-3-5272-1324 Fax: +81-3-5272-1208

zecca@aoni.waseda.jp (M. Zecca), takanisi@waseda.jp (A. Takanishi)

2. Italy-Japan joint laboratory on Humanoid and Personal Robotics

"RoboCasa", Tokyo, Japan

3. CRIM Lab - Scuola Superiore Sant'Anna, Pisa, Italy

4. EndoCAS - Center for Computer Assisted Surgery, Pisa, Italy

5. Graduate School of Science and Engineering, Waseda University, Tokyo

6. Department of Modern Mechanical Engineering, Waseda University, Tokyo, Japan

7. Humanoid Robotics Institute (HRI), Waseda University, Tokyo, Japan

8. Department of Mechanical Systems Engineering, Kogakuin University, Tokyo, Japan

9. Advanced Research Institute for Science and Engineering, Waseda University, Tokyo, Japan.,

Part of this research was conducted at the Humanoid Robotics Institute (HRI), Waseda University. The authors would like to express thanks to Okino Industries, LTD, Osada Electric Co., LTD, Sharp Corporation, Sony Corporation, Tomy Company, LTD, and ZMP, INC. for their financial support of HRI. And, the authors would like to thank the Italian Ministry of Foreign Affairs, General Directorate for Cultural Promotion and Cooperation, for its support for the establishment of the ROBOCASA laboratory. In addition, this research was supported by a Grant-in-Aid for the WABOT-HOUSE Project by Gifu Prefecture.

\section{INTRODUCTION}

$M$ IS has become the dominant technique in modern surgery as a consequence of the remarkable social and economic improvements that it provides. On the one hand, MIS procedures ensure many advantages to patients, while on the other hand, these procedures require surgeons to undergo a long and difficult training to manage and master them. In this context, a biomechanical analysis is crucial to establish efficient training exercises for enhancing the dexterity of surgeons and to define objective metrics for assessing the experience and performance of surgeons. Mainly, surgeons encounter both perceptual limitations (lack of stereoscopic view, limited field of view, and reduced force and tactile sensing) and motor limitations (reversed motion, movement scaling, and limited degrees-of-freedom) [1]. The skills required for MIS are very difficult to master because they are based on unique perceptual-motor relations. So, with the shift from open surgery to MIS, new problems have arisen related to the training of surgeons. Various training procedures have been established to enhance the dexterity of surgeons, and skill evaluation metrics have been developed to assess the experience and performance of surgeons.

Much of the previous work in the field of surgical training in virtual environments has been focused on the definition of metrics for an objective evaluation of surgical performance. One of the main objectives is to assess the abilities of surgeons and also to measure the skill levels of experts and novices. Many kinematic parameters and various indexes have been proposed for different surgical exercises [2-4]; in addition, segmentation procedures have been employed to characterize different phases of surgical movements [5, 6].

Our work is focused on assessing the abilities of surgeons in regard to coordination of bi-manual movements and depth perception during a typical laparoscopic exercise. Our aim is to define a set of parameters that allow us to characterize the surgeon's movements during a surgical procedure, to see how surgeons of different expertise rank in relation to these movement parameters, and to distinguish expert surgeons from less experienced surgeons such as residents. These analyses and modelling, in turn, represent a significant step towards the automatization and the robotic assistance of the surgical gesture.

Changes of human emotion appear in physiological 
parameters such as brain waves, respiration, heart rate, muscle tension, perspiration, pulse wave, and so on [7]. For example, heart rate increases when a human is surprised. On the other hand, except for reflex motions (e.g., in response to having one's eyelids touched or smelling ammonia), humans usually move their bodies with a particular intent. For example, humans square their shoulders if they are angry, reach out their hands to food if they are hungry, and so on. That is, the intent of human consciousness can be discovered by analyzing the motion of the head, eyes, arms, trunk, and hand grasp.

There already are many instruments on the market to measure the motion of the user (see Table 1). However, they are usually very expensive and/or not portable. Moreover, none of these systems is capable of measuring physiological parameters.

Table 1: Comparison of $W B-1 R$ with other motion- capture systems available on the market. UB: upper body; FB: full body.

\begin{tabular}{|c|c|c|c|c|c|}
\hline $\begin{array}{l}\underset{\infty}{\ll} \\
\stackrel{n}{\overrightarrow{0}} \\
\stackrel{B}{B}\end{array}$ & త్ర & 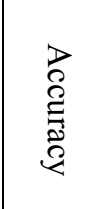 & 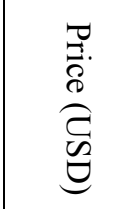 & 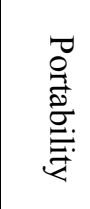 & 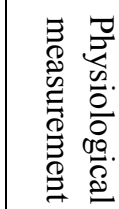 \\
\hline GypsyGyro18 & FB & High & 80,000 & Yes & No \\
\hline ShapeWrap II & FB & High & 26,500 & Yes & No \\
\hline VICON & FB & High & 47,250 & No & No \\
\hline WB-1R & UB & Low & 8,000 & Yes & Yes \\
\hline
\end{tabular}

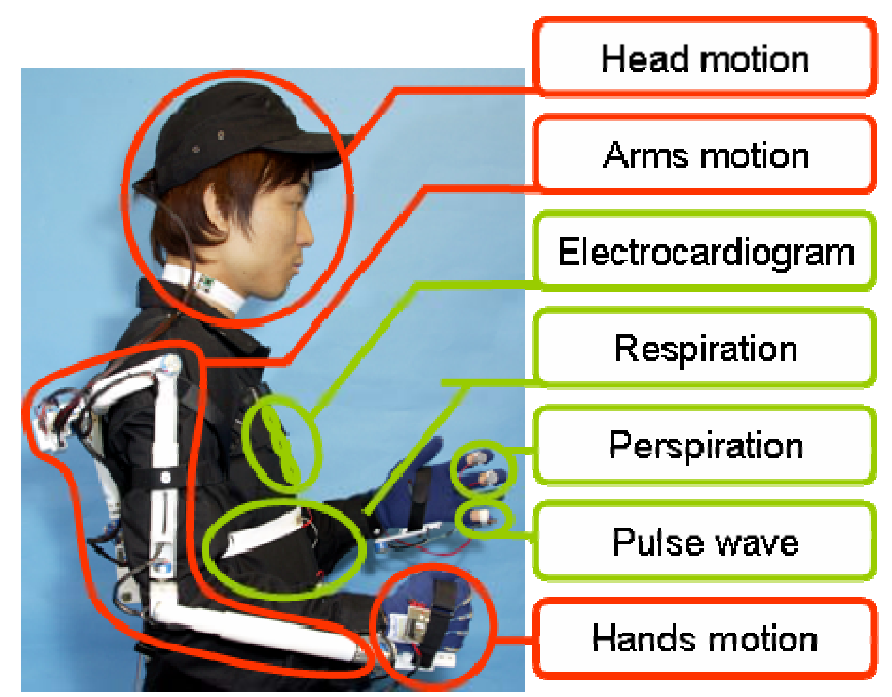

Fig. 1: The WB-1R. The motion-capture subsystem is in red; the physiological-parameters subsystem is in green.

In this paper we present the use of WB-1R (Waseda Bioinstrumentation system no. 1 Refined) $[8,9]$ as a possible tool to evaluate the experience and performance of surgeons during Laparoscopy. WB-1R consists of sensors that measure physiological parameters (heart rate, respiration, pulse wave, and perspiration) and a motion capture system that measures head motion, arm motion, and hand motion (Fig. 1). WB-1R can measure the movements and the physiological state of the user in real time. It weighs $2.2[\mathrm{~kg}]$, including batteries. The total system configuration is shown in Fig. 2.

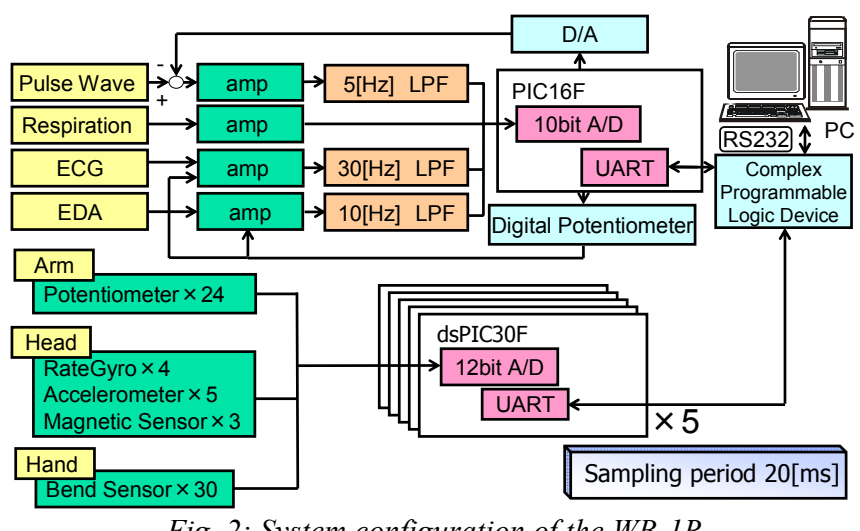

Fig. 2: System configuration of the $W B-1 R$.

\section{EXPERIMENTAL PROTOCOL}

The experimental protocol is the same as the one used in our previous work with the Optotrak [10]. In particular, we created a set of basic exercises to evaluate the surgeon's ability in regard to the handling of surgical instruments and depth perception using a laparoscopic view.

Seven students ( 5 males, 2 females) from the Faculty of Medicine at Pisa University and one expert surgeon ${ }^{1}$ (male) from the Department of Oncology of Pisa University gave their informed consent to participate in the experiment. Each subject performed the tests using a commercial virtual laparoscopic device, LapSIM Basic Skills 3.0 (Surgical Science AB, Sweden) and the Virtual Laparoscopic Interface (VLI) (Immersion Corporation, USA). The VLI allowed different subjects to execute identical exercises, which allowed us to elaborate generic and objective metrics independent of external variations; it also allowed the same subject to perform the same exercise at different times, which allowed us to monitor his/her learning curve [11, 12].

The users were asked to follow a standard training protocol that was developed during a European Lapsim Users Group meeting in 2005 in Utrecht. Based on virtual scenarios, the training protocol is structured around five consecutive repetitions of five different exercises focused on different abilities:

1. Navigation Instrument (NVI) - The subject has to reach, alternatively with the right and left tips of the instruments, some balls that appear one at a time;

2. Coordination (COO) - The subject controls the camera with one hand to find a ball, and then uses an instrument with the other hand to move towards the target balls that appear one at a time;

3. Grasping (GRA) - The subject has to reach, grasp, and stretch, alternatively with the right and left graspers of

\footnotetext{
${ }^{1}$ Although only one surgeon is not enough to get valid statistics, his data could be considered to be enough for this preliminary experiment. A more extensive analysis involving more subjects is currently ongoing, but the results are not yet available at this time.
} 
the instruments, some blood vessels that appear one at a time and then move them towards a target;

4. Lifting and Grasping $(L \& G)$ - The subject uses one instrument to lift an object with tissue-like properties and the other instrument to grasp it and move it toward a target ring placed below the object;

5. Cutting (CUT) - The subject has to grasp the end of a blood vessel with one instrument and cut it with the other instrument, an ultrasonic scissors triggered by a foot pedal.

Fig. 3 shows the laparoscopic simulator and the five different exercises.

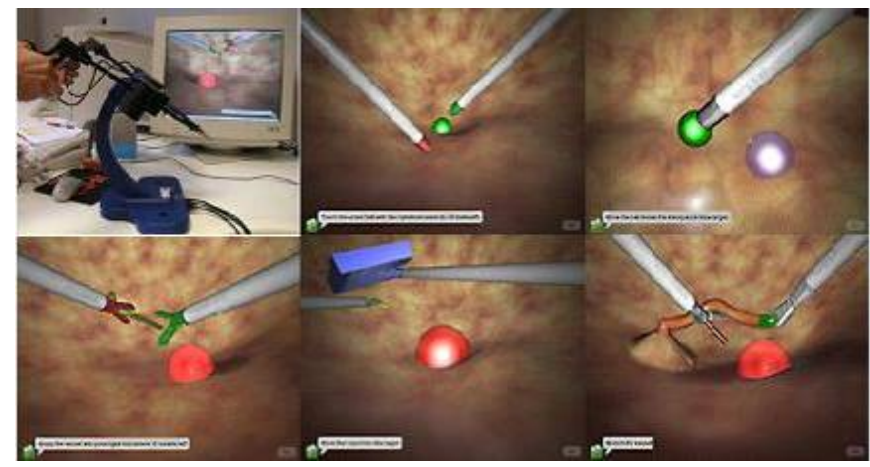

Fig. 3: From top left corner: The Virtual Laparoscopic Interface; Ex.\#1 Navigation Instrument (NVI); Ex.\#2 Coordination (COO); Ex.\#3 Grasping (GRA); Ex.\#4 Lifting and Grasping (L\&G); Ex.\#5 Cutting (CUT).

Fig. 4 shows the WB-1R worn by the surgeon during the experiments. Through the WB-1R worn by the subject, we were able to acquire data in regard to the motion of the head, both arms, and both hands, as well as data about heart beat, respiration, perspiration, and pulse wave. This data was acquired on a VAIO Laptop computer for real-time display, and saved for storage and offline analysis. All the data were saved as Comma Sparated Value, and they were analyzed in Matlab (The MathWorks, Inc.).

In a few cases the data of one of the subjects were affected by noise; so, they are not considered in the following analysis.

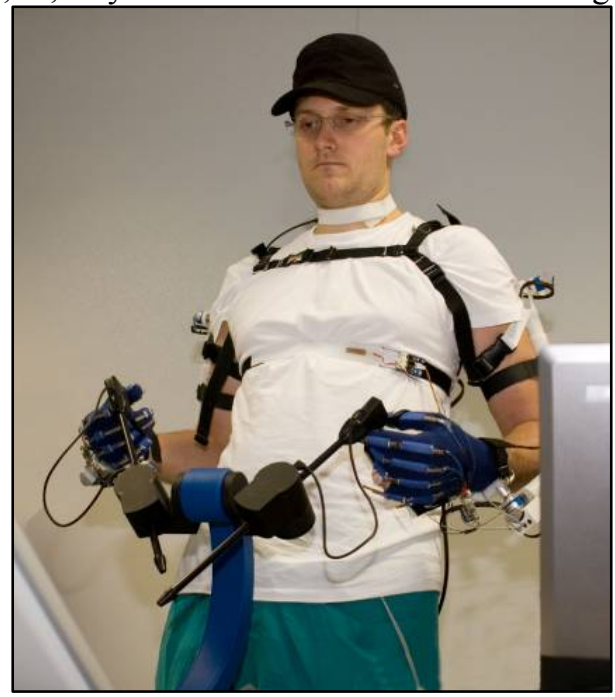

Fig. 4: The WB-1 worn by the expert surgeon

\section{EXPERIMENTAL EVALUATION}

In this paper we present the analysis and evaluation of three parameters:

A. comfort of the subjects while wearing the bioinstrumentation system during the simulations;

B. duration of the exercises (how long it took the subjects to complete each exercise);

C. respiration of the subjects during the simulations.

\section{A. Comfort of Subjects While Wearing the WB-1R}

At the end of the experimental protocol, each subject was asked if they experienced any particular problems using WB-1R. None of the subjects expressed any particular problems. However,

- two subjects (all students) reported that the Hand Motion Capture system slightly interfered with the movements of their hands while they were holding the laparoscopic simulator;

- two subjects (notably, the two female subjects) said that the system was slightly heavy; all the other subjects (all male) did not consider this to be a problem.

We will take these comments into account when we develop the next versions of WB-1R, so we can create a wearable bioinstrumentation system more suitable for the operating room.

\section{B. Duration of the exercises}

A first parameter to be analyzed is the duration of the exercises (i.e. the total time taken by subjects to complete each exercise). As already shown in [13], this parameter clearly enables identification of the expert surgeons and also characterization of the improvements made by novices over the entire experimental session. As expected, experts complete the exercises faster than the novices, and they also handle the instruments more smoothly. Also, as expected, experts improve less than novices.
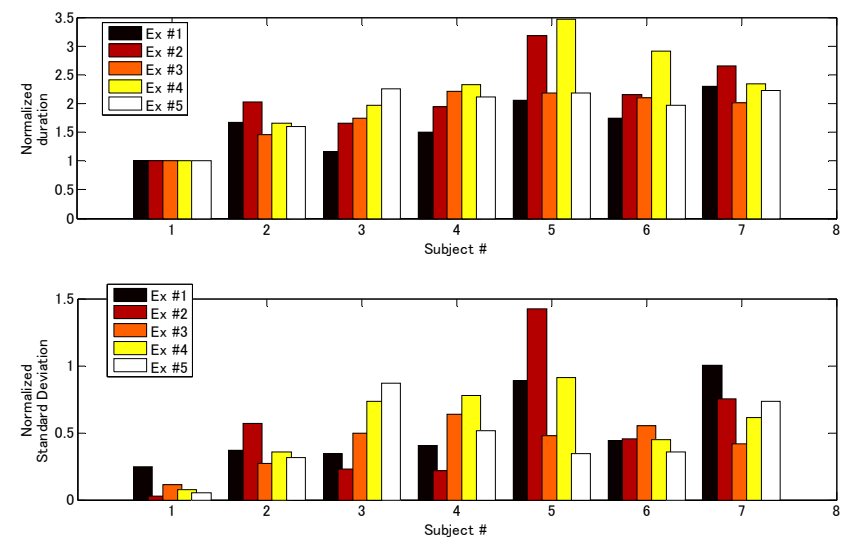

Fig. 5: Normalized durations and standard deviations for all exercises. Subject \#1 is the expert surgeon.

The time spent by subjects in each trial was useful to evaluate improvements in task performance (see Fig. 5 and Table 2). For the novices, the duration varied greatly, but the trend was for it to decrease from the first trial to the last. For 
the expert surgeon, the duration was quite constant, with a mean value lower than that of the novices, and the trend was for it to vary little from the first trial to the last. This confirms that experts make fewer (if any) improvements than novices.

It is also worth noting that the relatively great variation (normalized value: 0.24 ) for the surgeon in the first exercise is almost entirely due to the very first trial using the laparoscopic system. If this trial is not taken into account, the normalized standard deviation reduces to 0.11 , a value similar to that of the other exercises. We can reasonably assume similar variations for all the expert surgeons.

Table 2: Time taken by different subjects to complete the various exercises. Time is in sec.

\begin{tabular}{|c|c|c|c|c|c|c|c|c|}
\hline Ex\# & Series & $\begin{array}{c}\text { Novice } \\
\# 1\end{array}$ & $\begin{array}{c}\text { Novice } \\
\# 2\end{array}$ & Expert & $\begin{array}{c}\text { Novice } \\
\# 4\end{array}$ & $\begin{array}{c}\text { Novice } \\
\# 5\end{array}$ & $\begin{array}{c}\text { Novice } \\
\# 6\end{array}$ & $\begin{array}{c}\text { Novice } \\
\# 7\end{array}$ \\
\hline 1 & 1 & 45.36 & 38.96 & 32.21 & 49.23 & 81.23 & 53.72 & 92.46 \\
\hline 1 & 2 & 39.41 & 25.86 & 17.34 & 34.37 & 32.67 & 41.71 & 42.30 \\
\hline 1 & 3 & 28.81 & 19.23 & 21.44 & 31.78 & 36.48 & 39.37 & 43.24 \\
\hline 1 & 4 & & 27.03 & 21.66 & 29.92 & 40.94 & 35.69 & 39.29 \\
\hline 1 & 5 & & 20.59 & 20.59 & 24.57 & 40.14 & 26.18 & 42.27 \\
\hline \hline & $A V G$ & 37.86 & 26.33 & 22.65 & 33.97 & 46.29 & 39.33 & 51.91 \\
\hline & StDev & 8.38 & 7.80 & 5.62 & 9.25 & 19.80 & 9.99 & 22.71 \\
\hline
\end{tabular}

\begin{tabular}{|c|c|c|c|c|c|c|c|c|}
\hline Ex\# & Series & $\begin{array}{c}\text { Novice } \\
\# 1\end{array}$ & $\begin{array}{c}\text { Novice } \\
\# 2\end{array}$ & Expert & $\begin{array}{c}\text { Novice } \\
\# 4\end{array}$ & $\begin{array}{c}\text { Novice } \\
\# 5\end{array}$ & $\begin{array}{c}\text { Novice } \\
\# 6\end{array}$ & $\begin{array}{c}\text { Novice } \\
\# 7\end{array}$ \\
\hline 2 & 1 & 50.69 & 39.07 & 20.10 & 44.63 & 114.07 & 53.10 & 75.25 \\
\hline 2 & 2 & 43.33 & 37.46 & 19.62 & 38.77 & 53.90 & 53.15 & 61.32 \\
\hline 2 & 3 & 28.17 & 32.68 & 20.54 & 40.12 & 62.03 & 41.01 & 50.69 \\
\hline 2 & 4 & & 29.08 & 20.81 & 40.17 & 45.05 & 34.32 & 41.60 \\
\hline 2 & 5 & & 29.02 & 19.72 & 32.36 & 45.80 & 35.54 & 38.19 \\
\hline \hline & AVG & 40.73 & 33.46 & 20.16 & 39.21 & 64.17 & 43.42 & 53.41 \\
\hline & StDev & 11.49 & 4.66 & 0.52 & 4.42 & 28.74 & 9.21 & 15.15 \\
\hline
\end{tabular}

\begin{tabular}{|c|c|c|c|c|c|c|c|c|}
\hline Ex\# & Series & $\begin{array}{c}\text { Novice } \\
\# 1\end{array}$ & $\begin{array}{c}\text { Novice } \\
\# 2\end{array}$ & Expert & $\begin{array}{c}\text { Novice } \\
\# 4\end{array}$ & $\begin{array}{c}\text { Novice } \\
\# 5\end{array}$ & $\begin{array}{c}\text { Novice } \\
\# 6\end{array}$ & $\begin{array}{c}\text { Novice } \\
\# 7\end{array}$ \\
\hline 3 & 1 & 55.11 & 90.10 & 33.63 & 103.05 & 100.34 & 102.01 & 89.11 \\
\hline 3 & 2 & 56.47 & 55.24 & 41.00 & 94.93 & 81.09 & 78.01 & 79.40 \\
\hline 3 & 3 & 39.68 & 54.97 & 30.73 & 71.55 & 59.05 & 61.99 & 61.90 \\
\hline 3 & 4 & & 54.15 & 33.27 & 63.48 & 62.39 & 66.48 & 55.68 \\
\hline 3 & 5 & & 45.98 & 33.92 & 49.91 & 73.88 & 52.50 & 60.08 \\
\hline \hline & AVG & 50.42 & 60.09 & 34.51 & 76.58 & 75.35 & 72.20 & 69.24 \\
\hline & StDev & 9.32 & 17.21 & 3.84 & 22.05 & 16.53 & 19.02 & 14.31 \\
\hline
\end{tabular}

\begin{tabular}{|c|c|c|c|c|c|c|c|c|}
\hline Ex\# & Series & $\begin{array}{c}\text { Novice } \\
\# 1\end{array}$ & $\begin{array}{c}\text { Novice } \\
\# 2\end{array}$ & Expert & $\begin{array}{c}\text { Novice } \\
\# 4\end{array}$ & $\begin{array}{c}\text { Novice } \\
\# 5\end{array}$ & $\begin{array}{c}\text { Novice } \\
\# 6\end{array}$ & $\begin{array}{c}\text { Novice } \\
\# 7\end{array}$ \\
\hline 4 & 1 & 55.94 & 88.09 & 30.65 & 103.46 & 104.74 & 102.00 & 84.74 \\
\hline 4 & 2 & 50.53 & 65.15 & 27.14 & 71.67 & 129.99 & 87.24 & 87.08 \\
\hline 4 & 3 & 36.17 & 49.75 & 25.60 & 54.85 & 69.38 & 74.16 & 60.43 \\
\hline 4 & 4 & & 34.31 & 30.27 & 49.20 & 116.41 & 83.60 & 53.44 \\
\hline 4 & 5 & & 44.00 & 29.51 & 54.67 & 75.59 & 68.92 & 50.27 \\
\hline \hline & AVG & 47.54 & 56.26 & 28.63 & 66.77 & 99.22 & 83.18 & 67.19 \\
\hline & StDev & 10.22 & 21.02 & 2.17 & 22.18 & 26.08 & 12.80 & 17.50 \\
\hline
\end{tabular}

\begin{tabular}{|c|c|c|c|c|c|c|c|c|}
\hline Ex\# & Series & $\begin{array}{c}\text { Novice } \\
\# 1\end{array}$ & $\begin{array}{c}\text { Novice } \\
\# 2\end{array}$ & Expert & $\begin{array}{c}\text { Novice } \\
\# 4\end{array}$ & $\begin{array}{c}\text { Novice } \\
\# 5\end{array}$ & $\begin{array}{c}\text { Novice } \\
\# 6\end{array}$ & $\begin{array}{c}\text { Novice } \\
\# 7\end{array}$ \\
\hline 5 & 1 & 72.41 & 138.47 & 40.42 & 103.54 & 96.99 & 91.09 & 121.12 \\
\hline 5 & 2 & 52.35 & 77.83 & 36.16 & 84.57 & 81.62 & 79.63 & 99.55 \\
\hline 5 & 3 & 52.43 & 74.19 & 35.65 & 83.68 & 67.60 & 70.19 & 65.00 \\
\hline 5 & 4 & & 71.88 & 36.28 & 57.34 & 88.37 & 56.60 & 63.21 \\
\hline 5 & 5 & & 54.65 & 35.98 & 60.75 & 68.39 & 66.63 & 60.72 \\
\hline \hline & AVG & 59.06 & 83.40 & 36.90 & 77.98 & 80.59 & 72.83 & 81.92 \\
\hline & StDev & 11.56 & 32.05 & 1.98 & 19.05 & 12.73 & 13.12 & 27.08 \\
\hline
\end{tabular}

\section{Respiration of the Subjects}

Rubber bands with strain gauges (KFG-20-120-C1-11, Kyowa Electronic Instruments Co., Ltd.) were wound around the subject's abdomen and chest (Fig. 6). The output of the bridge circuit, which isneeded to reduce power consumption was amplified 10000-fold and processed by an A/D converter with a $20[\mathrm{~ms}]$ sampling period and a 10 [bit] resolution. After acquisition, 60 data measurements were then averaged with a moving average filter. If the interval of strain peak is denoted by $\Delta t_{r}$, the respiration rate $\mathrm{RR}$ is provided by $\mathrm{RR}=60 / \Delta t_{r}$ [bmp]. An example of the respiration data acquired during the experiments is shown in Fig. 7.

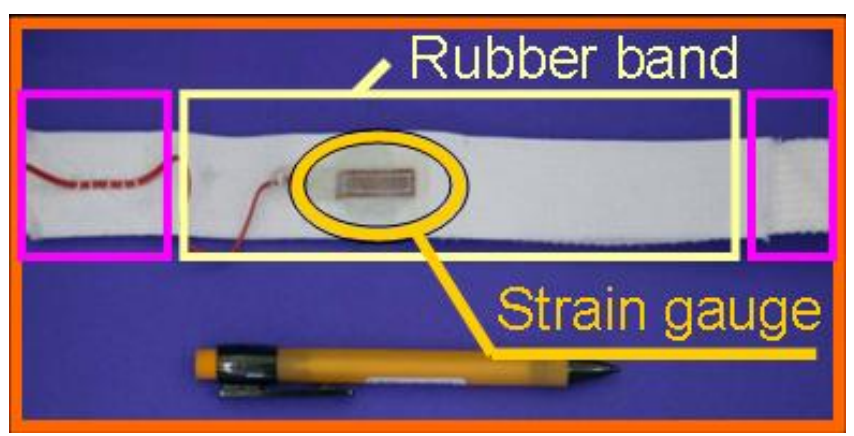

Fig. 6: Detail of the system used for the acquisition of the abdominal and thoracic respiration.

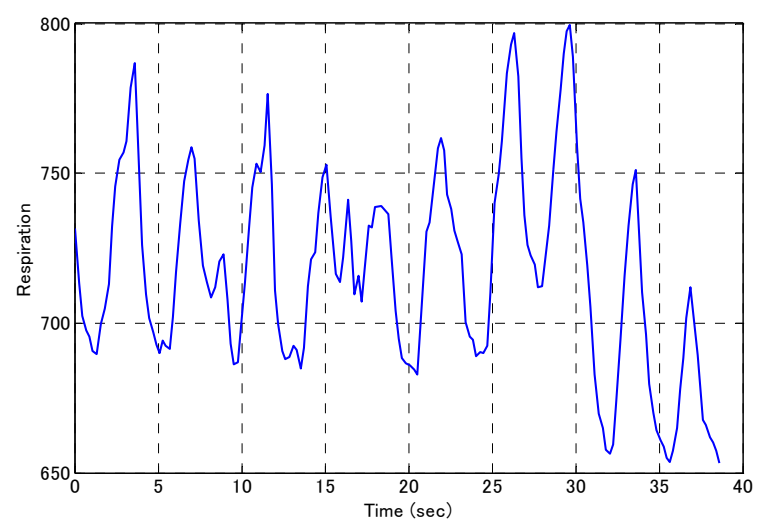

Fig. 7: One example of respiration data.

The respiration analysis of each subject for every exercise was computed in the frequency domain. The power spectral density of the abdominal chest signal was computed for each exercise of each subject. For this purpose, the PSD periodogram and the avgpower method were used to compute the average power of the signal. The results of this analysis are shown in the Fig. 8 through Fig. 12 for all the different exercises and all the subjects during the five trials.

As it can be seen in the next figures (Fig. 8-Fig. 12), the averaged power spectral density of the expert surgeon is significantly lower than that of the other subjects in all the exercises and in all the repetitions. 

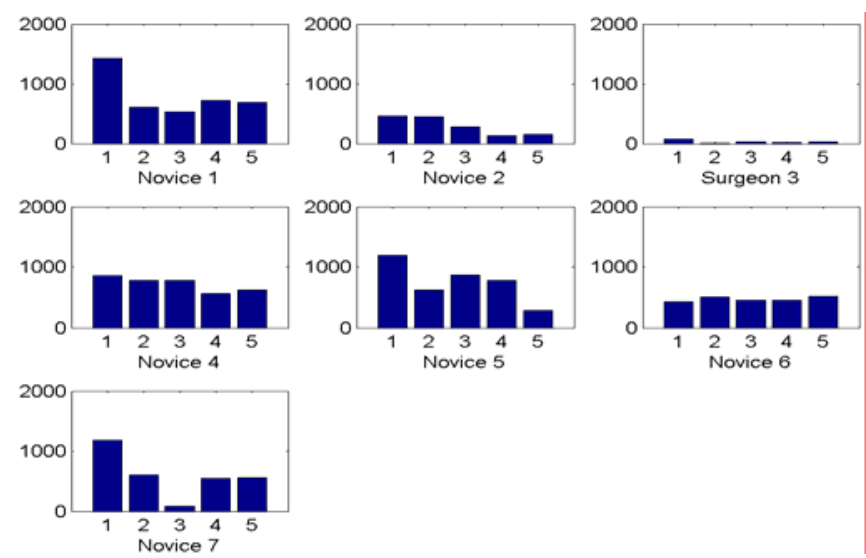

Fig. 8: Averaged power spectral density for exercise \#1.
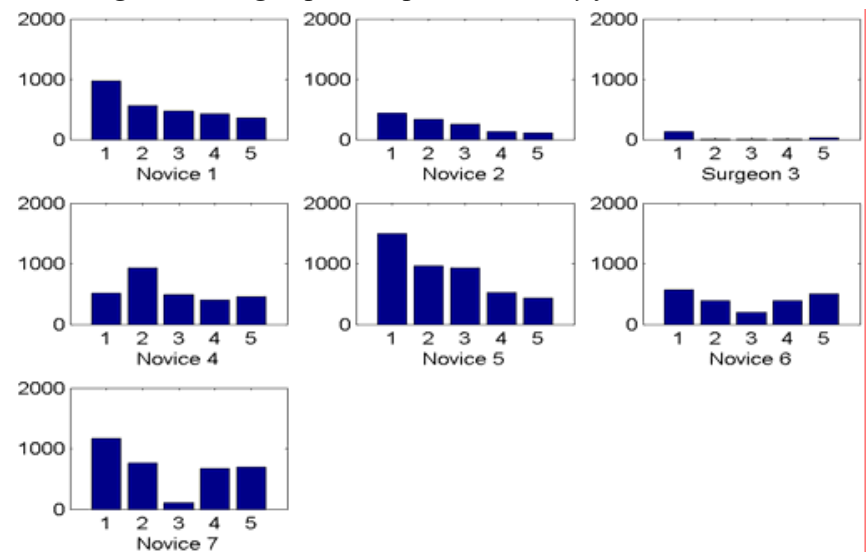

Fig. 9: Averaged power spectral density for exercise \#2.
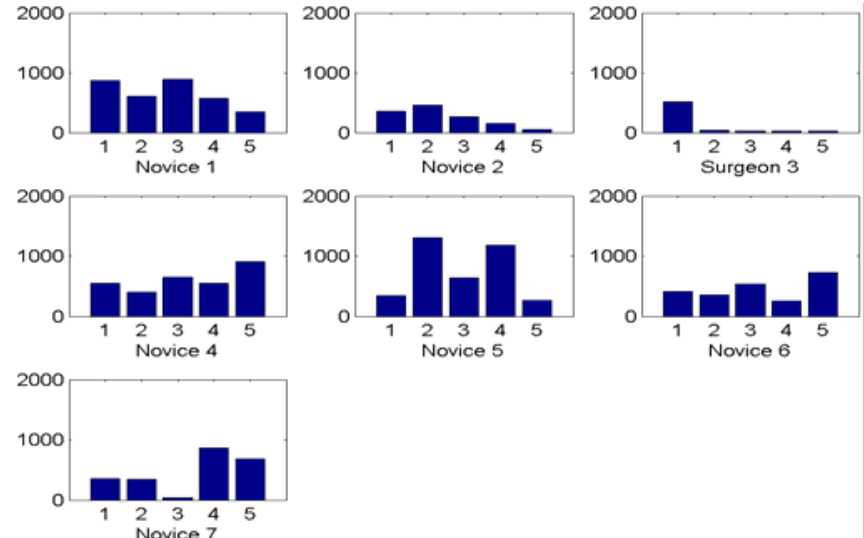

Fig. 10: Averaged power spectral density for exercise \#3.
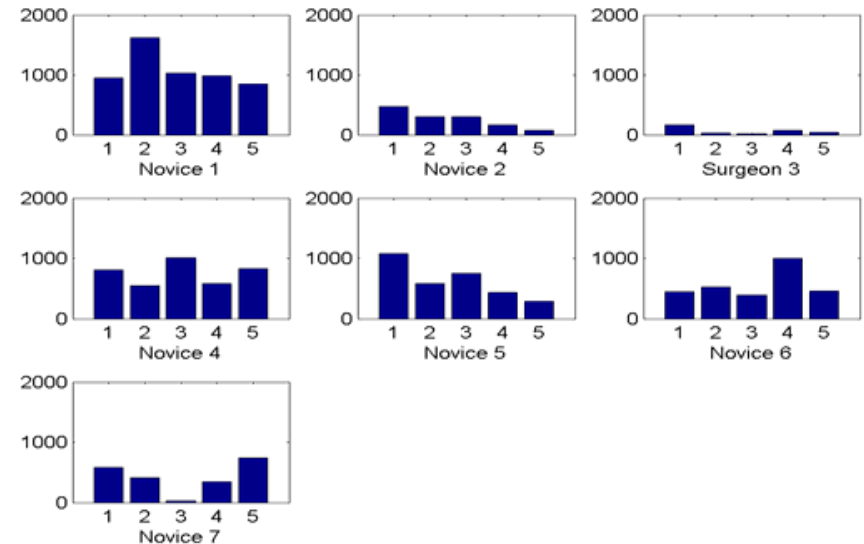

Fig. 11: Averaged power spectral density for exercise \#4.
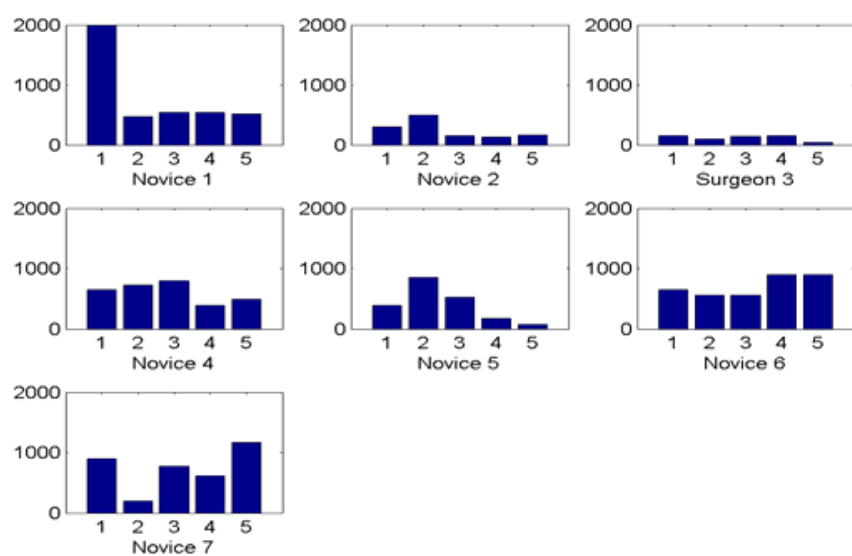

Fig. 12: Averaged power spectral density for exercise \#5.

Fig. 13 and Fig. 14 show the mean value of the averaged power spectral density (PSD) for each subject and each exercise. While there is no evident distinction among the five exercises (see Fig. 13), there is a clear difference between the expert surgeon and the other subjects (as clearly shown in Fig. 14). Incidentally, Novice \#2 seems to be the best subject in the group of non experts, as also shown in the previous section.The respiration, therefore, seems to be a good candidate for inclusion in a Global Performance Index. A more extensive analysis with more subjects is of course required.

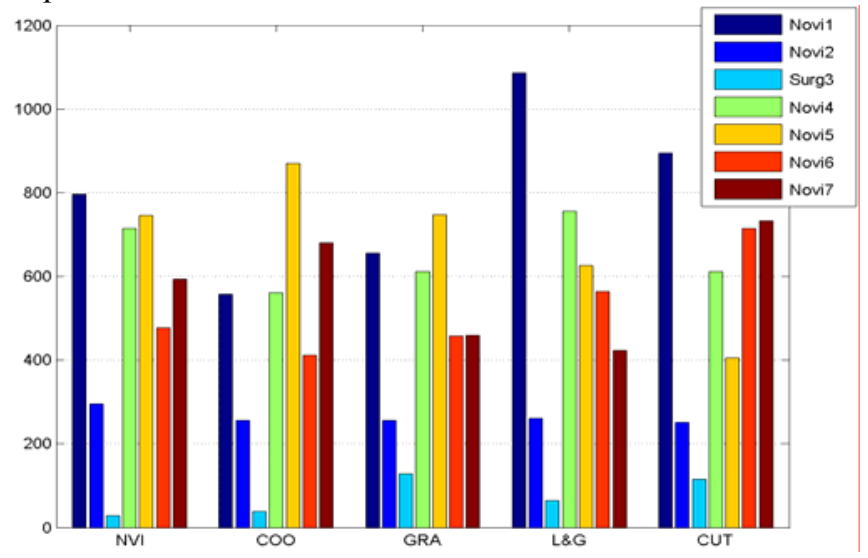

Fig. 13: Averaged power spectral density, grouped by exercise.

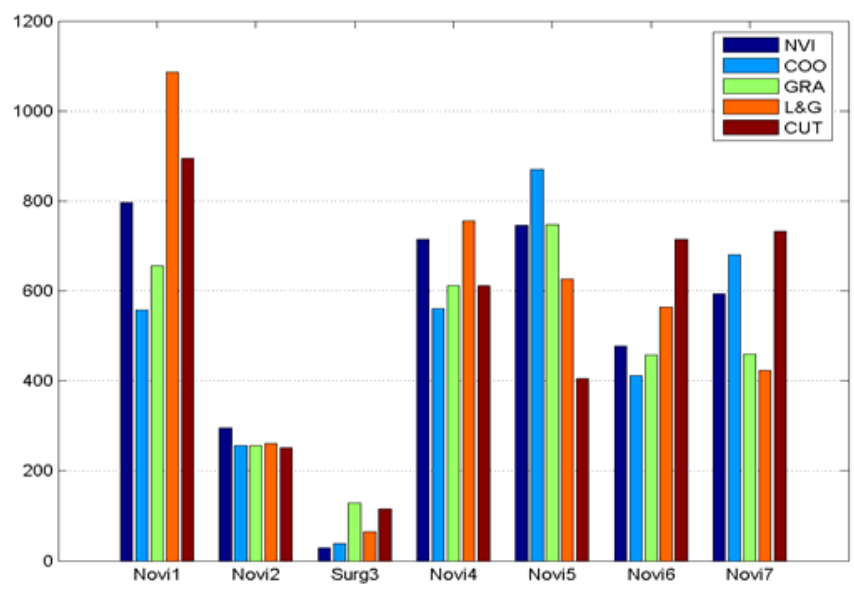

Fig. 14: Averaged power spectral density, grouped by subject. 


\section{CONCLUSION}

The dominance of MIS in modern medicine brings with it problems related to the training of surgeons. Training procedures have been established to enhance the dexterity of surgeons, and skill evaluation metrics have been developed to assess the experience and performance of surgeons.

In this paper, we discussed how we used the bioinstrumentation system WB-1R (Waseda $\underline{B}$ ioinstrumentation system No. $\underline{1}$ Refined) as a tool to assess the performance of surgeons during laparascopy. In particular, our goal was to assess the abilities of surgeons in regard to coordination of bi-manual movements and depth perception during a typical laparoscopic exercise. WB-1R was originally developed as an instrument for the objective analysis of human-robot interaction $[8,9]$. The advantage of this system is that it could be used as a measurement/validation tool not only during simulations but also during real surgery.

In these preliminary experiments, we collected the movements of the head, the arms, and the hands, as well as several physiological parameters (i.e. ECG, respiration, perspiration and pulse wave), for 8 subjects ( 1 experienced surgeon ant 7 students in medicine). Data of five consecutive repetitions of five different exercises focused on different abilities were acquired and stored.

In this paper, we presented a preliminary analysis of comfort, duration, and respiration. This analysis showed that the expert surgeon shows consistently high levels of ability during different trials and exercises; the group of novices, instead, displays varying levels of ability, and an overall worst performance (as expected).

Overall, this work shows that a substantial set of parameters is necessary to investigate and analyze the performance of surgeons. Currently, work is still in progress, and our future commitment in this field is to continue to analyze the performance of surgeons in more complex procedures and with other sensor systems. The next step would be to integrate this analysis with the processing of kinematic data describing movements of surgical instruments, as in [10]. Another possible step is the integration of WB-1R (or, better, its future version) with other evaluation systems, like the one proposed in [14].

The results presented in this paper are a clear step towards the development of a a training system for an objective evaluation of the surgeon's performance, in particular during MIS. These results could also be extended to develop instruments and methodologies for a functional/ergonomic evaluation of surgical instruments. These analysis and modeling, moreover, are an important step towards the automatization and the robotic assistance of the surgical gesture.

\section{ACKNOWLEDGMENT}

The authors would also like to express their thanks to the following for their support of our research: ARTS Lab; NTT Docomo; Solid Works Corp.; Consolidated Research
Institute for Advanced Science and Medical Care, Waseda University; Advanced Research Institute for Science and Engineering, Waseda University; Prof. Yutaka Kimura, Dr. Yuichiro Nagano and Dr. Naoko Yoshida for their support for our research. Eventually, the authors would like to thank Prof. Robert DiGiovanni for his proofreading of the manuscript and his suggestions.

\section{REFERENCES}

[1] Shah and Darzi, "The impact of inherent and environmental factors on surgical performance in laparoscopy: a review," Minim Invasive Ther Allied Technol, vol. 12, pp. 69-75, Mar 2003.

[2] S. Cotin, N. Stylopoulos, M. Ottensmeyer, P. Neumann, D. Rattner, and S. Dawson, "Metrics for Laparoscopic Skills Trainers: The Weakest Link!," in Medical Image Computing and Computer-Assisted Intervention - MICCAI 2002: 5th International Conference, Tokyo, Japan, September 25-28, 2002, Proceedings, Part I, 2002, pp. 35-43.

[3] C. G. L. Cao and C. L. MacKenzie, "Task and motion analyses in endoscopic surgery," in ASME IMECE Conference Proceedings: 5th Annual Symposium on Haptic Interfaces for Virtual Environment and Teleoperator Systems, Atlanta, Georgia, 1996, pp. 583--590.

[4] L. Verner, D. Oleynikov, S. Holtmann, H. Haider, and L. Zhukov, "Measurements of the level of surgical expertise using flight path analysis from da VinciTM robotic surgical system," in Medicine Meets Virtual Reality, 2003, pp. 373-378.

[5] D. Risucci, J. A. Cohen, J. E. Garbus, M. Goldstein, and M. G. Cohen, "The effects of practice and instruction on speed and accuracy during resident acquisition of simulated laparoscopic skills," Curr Surg, vol. 58, pp. 230-235, Mar 2001.

[6] S. Payandeh, A. Lomax, J. Dill, C. Mackenzie, and C. G. L. Cao, "On Defining Metrics for Assessing Laparoscopic Surgical Skills in a Training Envirnoment," in Studies in Health Technology and Informatics - Medicine Meets Virtual Reality, 2002, pp. 334--340.

[7] Japanese Society of Medical Electronics and Biological Engineering Iyou Denshi Seitai Kougaku, "ME Jiten (in Japanese)," Corona Publishing CO., LTD 1978.

[8] K. Itoh, H. Miwa, Y. Nukariya, M. Zecca, H. Takanobu, S. Roccella, M. C. Carrozza, P. Dario, and A. Takanishi, "Development of a Bioinstrumentation System in the Interaction between a Human and a Robot," in Intelligent Robots and Systems, 2006 IEEE/RSJ International Conference on, 2006, pp. 2620-2625.

[9] M. Zecca, N. Endo, K. Itoh, K. Imanishi, M. Saito, N. Nanba, H. Takanobu, and A. Takanishi, "On the development of the Bioinstrumentation System for the evaluation of human-robot interaction - Head and Hands Motion Capture Systems," in 2007 IEEE/ASME International Conference on Advanced Intelligent Mechatronics, ETH Zürich, Switzerland, 2007, p. in press.

[10] G. Megali, S. Sinigaglia, O. Tonet, and P. Dario, "Modelling and evaluation of surgical performance using hidden Markov models," IEEE Trans Biomed Eng, vol. 53, pp. 1911-9, Oct 2006.

[11] D. T. Woodrum, P. B. Andreatta, R. K. Yellamanchilli, L. Feryus, P. G. Gauger, and R. M. Minter, "Construct validity of the LapSim laparoscopic surgical simulator," Am J Surg, vol. 191, pp. 28-32, Jan 2006.

[12] K. W. van Dongen, E. Tournoij, D. C. van der Zee, M. P. Schijven, and I. A. Broeders, "Construct validity of the LapSim: can the LapSim virtual reality simulator distinguish between novices and experts?," Surg Endosc, vol. 21, pp. 1413-7, Aug 2007.

[13] F. Cavallo, G. Megali, S. Sinigaglia, O. Tonet, P. Dario, and A. Pietrabissa, "A biomechanical analysis of bi-manual coordination and depth perception in virtual laparoscopic surgery," in Computer Assisted Radiology and Surgery (CARS) - 10th Annual Conference of the International Society for Computer Aided Surgery, 2006.

[14] N. Oshima, J. Solis, Y. Ogura, K. Hatake, and A. Takanishi, "Design of a New Suture/Ligature Evaluation System WKS-2," in 2007 IEEE/RSJ International Conference on Intelligent Robots and Systems, Sheraton Hotel, San Diego, CA, USA, 2007, p. in press. 